\title{
An update of mortality among chemical workers exposed to benzene
}

\author{
G G BOND, E A MCLAREN, C L BALDWIN, R R COOK \\ From Dow Chemical USA, Epidemiology, $H \&$ ES, Midland, Michigan 48674, USA
}

ABSTRACT Mortality was updated to the end of 1982 for 594 employees exposed to benzene who had been studied previously and for an additional 362 exposed workers not studied previously. Cause specific mortality comparisons were made using United States white male, age, and calendar year adjusted rates. Total mortality was observed to have been significantly below expectation, and this was particularly evident for deaths from accidental causes. Mortality from skin cancer was significantly raised, although there were no unusual or common characteristics among the affected individuals which would suggest a link with exposure to benzene. A non-significant excess of total deaths from leukaemia was noted based on four observed cases; however, all four were myelogenous leukaemias and this represented a significant excess in that subcategory. These and other deaths of possible interest are reviewed in detail. Analyses by work area, duration of exposure, and cumulative dose index did not show patterns suggestive of a causal association between exposure to benzene and any particular cause of death.

There is a large amount of evidence to support the hypothesis that acute myelocytic leukaemia may be a latent effect of overexposure to benzene ${ }^{1}$ (B D Goldstein, unpublished data). Data to support increased risks of other types of leukaemia or other cancers have been reported, but are in need of further evaluation ${ }^{23}$ (O Wong, unpublished data). The exact shape of the dose response curve for the benzene leukaemia association is unknown and has been the source for speculation and controversy. ${ }^{145}$ One of the few studies which has been relied on for risk assessment was reported by Ott et al in 1978. ${ }^{6}$ They followed 594 Dow, Michigan Division, employees potentially exposed in any of three production areas using benzene. Two deaths from leukaemia were observed compared with one expected to the end of 1973. After excluding cohort members with other hazardous exposure, they observed one death from leukaemia against 0.9 expected. A third subject died of bilateral bronchopneumonia but had myeloblastic leukaemia listed on the death certificate under "other significant conditions." This death was coded to pneumonia consistent with the rules followed by the World Health Organisation. The present study updates the mortality experience of that cohort of 594 with an additional nine years of follow up to the end of 1982, and

Accepted 3 February 1986 includes 362 exposed employees not studied originally.

\section{Methods and materials}

A detailed description of the chlorobenzol, alkyl benzene, and ethyl cellulose operations in which these employees had potential exposure to benzene was given by Ott et al. ${ }^{6}$ That report also should be consulted for summaries of industrial hygiene measurements used in exposure classification, and the methods by which the cohort was identified.

In brief, 594 employees were identified from annual census lists as having worked for one month or longer between 1938 and 1970 at jobs of interest in the three production areas. For the present study, the census lists were consulted again. This included a review of monthly census lists available beginning in 1965 . These had not been used for the original study. An additional 228 employees who met the cohort definition of Ott $e t a l^{6}$ were identified by this review and are included in the present investigation. Monthly census lists from 1970 to 1978 were consulted to ascertain another 134 employees potentially exposed after the closing date of the original study. Thus the present study reports mortality among 956 employees.

All job assignments involving potential exposure to benzene to the end of 1978 were coded and considered 
for exposure classification. Each job entry was assigned one of four exposure intensity levels (very low, low, moderate, and high) based on available industrial hygiene monitoring data. All jobs held after 1972 were classified as either low or very low exposure.

A cumulative dose index was calculated for each cohort member by multiplying the representative TWA value for each intensity level by the number of days exposed at each level, and dividing by 30.41 days/month to yield ppm months. As in the original paper of Ott et al, ${ }^{6}$ the TWA value of $1 \mathrm{ppm}$ was used for the very low level, $5 \mathrm{ppm}$ for low level, $17 \mathrm{ppm}$ for moderate level, and $30 \mathrm{ppm}$ for high level.

Follow up of the cohort for vital status determination was done using company records, the earnings and benefits records of the Social Security Administration to the end of 1979 , and the National Death Index to the end of 1982. Death certificates were obtained and were coded according to the International Classification of Diseases (ICD) rules in effect at the time of death by a nosologist experienced in multiple revision coding. Deaths were then tabulated by cause for analysis using the categories of the 8th ICD revision.

Analyses were carried out by production area, duration of exposure, and by cumulative dose index. Employees may have contributed person-years and deaths to several categories. Allowance was made for a possible latency period by lagging exposures 15 years. Expected deaths were calculated by the indirect method using United States white male mortality rates via an updated version of the Monson programme (USDR 58). ${ }^{7}$ Standardised mortality ratios (SMRs) were calculated as the ratio of observed to expected deaths multiplied by 100 , and Fisher's exact $95 \%$ confidence limits about the point estimates ${ }^{8}$ were computed using the programmes of Rothman and Boice. ${ }^{9}$ Examination of trends across SMRs was done using a $\chi^{2}$ trend test with one degree of freedom as outlined by Breslow et al. ${ }^{10}$

Previous studies at this plant had identified employees with past exposure to arsenic, ${ }^{11}$ asbestos, ${ }^{12}$ or high levels of vinyl chloride ${ }^{13}$ as possibly being at increased risk for several malignancies. Analyses were made both including and excluding those employees from the present cohort.

\section{Results}

Table 1 shows the vital and employment status distribution of the cohort on 31 December 1982. Follow up was $98.4 \%$ complete; the 15 employees lost to follow up were withdrawn from the life table on the date they were last known to have been alive. A total of 225 deaths was identified, 25 of which were among the 68 employees who also had been exposed to asbestos, arsenic or vinyl chloride. Death certificates were obtained for all but one $(0.4 \%)$ of the decedents; this death of unknown cause was included in the totals for analysis but was not allocated to any cause specific category.

The distribution of employees by period of first exposure and duration of exposure is presented in table 2. The median year of first exposure was 1948, and $78.6 \%$ of cohort members were first exposed at least 15 years before the closing date of the study. The mean duration of exposure was 7.0 years (median = 2.6). The 956 employees contributed a total of 24571 person years, for an average follow up of 25.7 years. The average age at entry into follow up was 31.0 years.

Table 3 presents the distribution of cohort members by exposure intensity level, and within each level by year of first exposure and duration of exposure Improvements in exposure conditions over time anto job mobility by employees resulted in most having been exposed at more than one intensity level. Nearly a quarter (229 subjects) of the cohort members were exposed in jobs categorised as 30 ppm TWA benzene exposure, and nearly a third (311) were exposed in jobs categorised as $17 \mathrm{ppm}$ TWA.

Table 4 shows the overall mortality comparisons for selected causes of death. The all causes SMR for this cohort was 84 (or 83 if the arsenic, asbestos, and vinyl chloride exposed subset is excluded). This represented a significant deficit compared with expectation but was consistent with the general pattern of lower mortality among employees from this plant. ${ }^{12} \mathrm{~A}$

Table 1 Vital and employment status distribution of benzene cohort on 31 December 1982

\begin{tabular}{|c|c|c|c|c|}
\hline Vital and employment status & Total cohort & (\% of total) & Less exclusions* & $(\%$ of total $)$ \\
\hline $\begin{array}{l}\text { Total population } \\
\text { Still working } \\
\text { Retired } \\
\text { Dead (company records) } \\
\text { Left company: } \\
\text { Dead } \\
\text { Living } \\
\text { Unknown }\end{array}$ & $\begin{array}{r}956 \\
197 \\
273 \\
155 \\
331 \\
70 \\
246 \\
15\end{array}$ & $\begin{array}{r}(100 \cdot 0) \\
(20 \cdot 6) \\
(28 \cdot 6) \\
(16 \cdot 2) \\
(34 \cdot 6) \\
(7 \cdot 3) \\
(25 \cdot 7) \\
(1 \cdot 6)\end{array}$ & $\begin{array}{r}888 \\
192 \\
246 \\
135 \\
315 \\
65 \\
235 \\
15\end{array}$ & $\begin{array}{r}(100 \cdot 0) \\
(21 \cdot 6) \\
(27 \cdot 7) \\
(15 \cdot 2) \\
(35 \cdot 5) \\
(7.3) \\
(26.5) \\
(1.7)\end{array}$ \\
\hline
\end{tabular}

*Less those exposed to arsenic, asbestos, or high levels of vinyl chloride (see text). 
Table 2 Distribution of benzene cohort by year of first exposure and duration of exposure

\begin{tabular}{lrrrrr}
\hline $\begin{array}{l}\text { Calendar } \\
\text { period of } \\
\text { first exposure }\end{array}$ & \multicolumn{5}{l}{ Duration of exposure (years)* } \\
\cline { 2 - 6 } & $<1$ & $1-4.9$ & $5-14.9$ & $\geqslant 15$ & Total \\
\hline$<1940$ & 6 & 39 & 37 & 55 & 137 \\
$1940-49$ & 106 & 122 & 47 & 76 & 351 \\
$1950-59$ & 35 & 45 & 38 & 23 & 141 \\
$1960-69$ & 72 & 53 & 60 & 4 & 189 \\
$1970-78$ & 46 & 74 & 18 & 0 & 138 \\
Total & 265 & 333 & 200 & 158 & 956 \\
\hline
\end{tabular}

*Adjusted for breaks in service and accumulated to the end of 1978 .

significant excess was observed for skin cancer based on four observed deaths. A substantial and significant deficit was observed for the category of accidental deaths. No other significant results were found for the other 54 causes of death evaluated.

Four deaths were observed from leukaemia, resulting in a non-significant excess for this category. The case summaries for these four deaths and for a fifth case classified to pneumonia are given in table 5 . Case numbers 1-3 were originally reported by Ott $e t$ al. ${ }^{6}$ Case number 4 died in 1980 after the original follow up ended. This man began work at the company in 1943 as an operator in Saran fabrication, shortly thereafter he moved to alkyl benzene where he held several operator assignments with potential high level benzene exposure until he retired in 1962. His estimated career dosage was $4211 \mathrm{ppm}$ months. He died aged 80 of myelomonocytic leukaemia; no necropsy was performed. The interval between the onset of disease and death was reported to have been two months.

Case number 5 was not included in the original Ott cohort. He began work with the company in 1928 and worked for one month in an area of potential arsenic exposure before moving to chlorobenzol where he worked first as an operator then as a shift foreman in jobs classified as low or very low benzene exposure until his retirement in 1952. His estimated career dosage was 343 ppm months. He died in 1967 aged 80 of acute myelogenous leukaemia.

As was true for the three deaths from leukaemia observed by Ott et al, both the new leukaemias had the potential for unquantified brief, but potentially relatively high, exposures to benzene. In the alkyl benzene area, benzene levels were measured as high as 283 ppm in some samples; in ethyl cellulose, as high as 937 ppm. Therefore, caution must be exercised when interpreting the cumulative dose indices.

There was a non-significant excess of deaths observed in the category of other and unspecified cancers. A review of the death certificate diagnoses for the seven affected individuals did not show a common tumour type nor any that were of lymphatic tissue.

Table 3 Distribution of benzene cohort* by exposure intensity level and within by year of first exposure and duration

\begin{tabular}{|c|c|c|c|c|c|c|c|}
\hline \multirow{2}{*}{\multicolumn{2}{|c|}{ Intensity level and year of first exposure }} & \multicolumn{6}{|c|}{ Duration (years) $\dagger$} \\
\hline & & \multirow{2}{*}{$\begin{array}{r}<1 \\
261 \\
22 \\
85 \\
45 \\
33 \\
76\end{array}$} & \multirow{2}{*}{$\begin{array}{r}1- \\
54 \\
4 \\
13 \\
12 \\
5 \\
20\end{array}$} & \multirow{2}{*}{$\begin{array}{r}2- \\
53 \\
3 \\
10 \\
6 \\
22 \\
12\end{array}$} & \multirow{2}{*}{$\begin{array}{r}5- \\
48 \\
6 \\
11 \\
11 \\
15 \\
5\end{array}$} & \multirow{2}{*}{$\begin{array}{c}10- \\
49 \\
16 \\
15 \\
10 \\
8 \\
-\end{array}$} & \multirow{2}{*}{$\begin{array}{r}\text { Total } \\
465 \\
51 \\
134 \\
84 \\
83 \\
113\end{array}$} \\
\hline Very low & $\begin{aligned} & \text { Total } \\
< & 1940 \\
& 1940-9 \\
& 1950-9 \\
& 1960-9 \\
\geqslant & 1970\end{aligned}$ & & & & & & \\
\hline Low & $\begin{aligned} & \text { Total } \\
&< 1940 \\
& 1940-9 \\
& 1950-9 \\
& \\
& 1960-9 \\
& \geqslant 1970\end{aligned}$ & $\begin{array}{r}476 \\
28 \\
165 \\
58 \\
117 \\
108\end{array}$ & $\begin{array}{r}55 \\
2 \\
16 \\
6 \\
21 \\
10\end{array}$ & $\begin{array}{r}98 \\
8 \\
26 \\
16 \\
27 \\
21\end{array}$ & $\begin{array}{r}50 \\
7 \\
22 \\
12 \\
9 \\
-\end{array}$ & $\begin{array}{r}54 \\
15 \\
27 \\
-9 \\
3 \\
-\end{array}$ & $\begin{array}{r}733 \\
60 \\
256 \\
101 \\
177 \\
139\end{array}$ \\
\hline Moderate & $\begin{aligned} & \text { Total } \\
&< 1940 \\
& 1940-9 \\
& 1950-9 \\
& 1960-9 \\
& 1960\end{aligned}$ & $\begin{array}{r}203 \\
19 \\
102 \\
25 \\
38 \\
19\end{array}$ & $\begin{array}{r}24 \\
3 \\
7 \\
11 \\
3 \\
\end{array}$ & $\begin{array}{r}37 \\
17 \\
11 \\
5 \\
4 \\
-\end{array}$ & $\begin{array}{r}15 \\
3 \\
6 \\
5 \\
1 \\
-\end{array}$ & $\begin{array}{r}32 \\
5 \\
25 \\
2 \\
-\end{array}$ & $\begin{array}{r}311 \\
47 \\
151 \\
48 \\
46 \\
19\end{array}$ \\
\hline High & $\begin{aligned} & \text { Total } \\
< & 1940 \\
& 1940-9 \\
& 1950-9 \\
& 1960-9 \\
\geqslant & 1970\end{aligned}$ & $\begin{array}{r}133 \\
13 \\
96 \\
11 \\
13 \\
-\end{array}$ & $\begin{array}{r}30 \\
4 \\
21 \\
5 \\
-\end{array}$ & $\begin{array}{r}31 \\
5 \\
21 \\
5 \\
-\end{array}$ & $\begin{array}{r}25 \\
4 \\
15 \\
6 \\
- \\
-\end{array}$ & $\begin{array}{r}10 \\
4 \\
5 \\
1 \\
- \\
-\end{array}$ & $\begin{array}{r}229 \\
30 \\
158 \\
28 \\
13 \\
\end{array}$ \\
\hline
\end{tabular}

*Employees may have been exposed at more than one level and therefore may be counted more than once in the table.

tAdjusted for breaks in service and accumulated to the end of 1978. 
Table 4 Observed and expected deaths by cause, SMRs, and 95\% confidence limits. ${ }^{*}$ Mortality among benzene cohort, 1940-82

\begin{tabular}{|c|c|c|c|c|c|c|c|c|}
\hline \multirow[b]{2}{*}{ Cause of death (ICD-8th) } & \multicolumn{4}{|c|}{ Total cohort } & \multicolumn{4}{|c|}{ Cohort less exclusions $\dagger$} \\
\hline & Obs & Exp & $S M R$ & $95 \% C I^{*}$ & Obs & $\operatorname{Exp}$ & $S M R$ & $95 \% C I^{*}$ \\
\hline \multirow{4}{*}{$\begin{array}{l}\text { All causes (including } 1 \text { unknown) } \\
\text { All malignant neoplasms }(140-209) \\
\text { Buccal cavity of pharynx }(140-149) \\
\text { Digestive organs and peritoneum } \\
(150-159)\end{array}$} & 225 & $268 \cdot 6$ & 84 & $73-95$ & 200 & $242 \cdot 3$ & 83 & $72-95$ \\
\hline & 59 & $54 \cdot 7$ & 108 & $82-139$ & 49 & $49 \cdot 2$ & 100 & $74-132$ \\
\hline & 2 & 1.8 & 111 & $13-401$ & 2 & 1.6 & 124 & $15-452$ \\
\hline & 13 & 15.1 & 86 & $46-147$ & 12 & 13.6 & 89 & $46-154$ \\
\hline Oesophagus $(150)$ & $\begin{array}{r}13 \\
0\end{array}$ & $\begin{array}{r}1.1 \\
1.3\end{array}$ & $\stackrel{80}{-}$ & $\begin{array}{r}40-141 \\
0-284\end{array}$ & $\begin{array}{r}12 \\
0\end{array}$ & $\begin{array}{r}130 \\
1.2\end{array}$ & $\underline{89}$ & $\begin{array}{r}40-134 \\
0-307\end{array}$ \\
\hline Stomach (151) & 3 & 2.8 & 106 & $22-313$ & 3 & $2 \cdot 5$ & 118 & $25-351$ \\
\hline Large intestine (153) & 7 & 4.8 & 145 & $59-300$ & 6 & $4 \cdot 4$ & 138 & $50-297$ \\
\hline Rectum (154) & 0 & 1.7 & - & $0-217$ & 0 & 1.5 & - & $0-246$ \\
\hline Liver $(155-156)$ & 0 & $1 \cdot 1$ & - & $0-335$ & 0 & 1.0 & - & $0-369$ \\
\hline Pancreas (157) & 2 & 3.0 & 67 & $8-241$ & 2 & $2 \cdot 7$ & 75 & $9-268$ \\
\hline Respiratory system $(160-163)$ & 20 & $19 \cdot 1$ & 104 & $64-162$ & 14 & $17 \cdot 2$ & 81 & $45-137$ \\
\hline Larynx (161) & 0 & 0.8 & - & $0-461$ & 0 & 0.8 & - & $0-461$ \\
\hline Lung $(162-163)$ & 18 & $18 \cdot 1$ & 99 & $59-157$ & 13 & $16 \cdot 3$ & 80 & $42-136$ \\
\hline Bone (170) & 0 & $0 \cdot 3$ & - & $0-1230$ & 0 & 0.2 & - & $0-1845$ \\
\hline Skin $(172-173)$ & 4 & $1 \cdot 0$ & 400 & $109-1024$ & 4 & 0.9 & 441 & $121-1138$ \\
\hline Prostate $(185)$ & 3 & $3 \cdot 2$ & 95 & $19-274$ & 2 & $2 \cdot 8$ & 70 & $9-258$ \\
\hline Testis (186) & 0 & 0.3 & - & $0-1230$ & 0 & 0.3 & - & $0-1230$ \\
\hline Bladder (188) & 1 & 1.5 & 66 & $2-371$ & 0 & 1.4 & - & $0-264$ \\
\hline Kidney (189) & 1 & $1 \cdot 4$ & 72 & $2-398$ & 1 & $1 \cdot 3$ & 80 & $2-429$ \\
\hline Eye $(190)$ & 0 & 0.1 & - & $0-3689$ & 0 & 0.0 & - & - \\
\hline Brain and other nervous systems & & & & & & & & \\
\hline$(191-192)$ & 1 & $1 \cdot 7$ & 58 & $1-328$ & 1 & 1.6 & 64 & $2-348$ \\
\hline Thyroid (193) & 0 & $0 \cdot 1$ & - & $0-3689$ & 0 & 0.1 & - & $0-3689$ \\
\hline Lymphosarcoma and & & & & & & & & \\
\hline reticulosarcoma (200) & 2 & $1 \cdot 1$ & 180 & $22-657$ & 2 & $1 \cdot 0$ & 199 & 24-722 \\
\hline Hodgkin's disease (201) & 0 & 0.7 & - & $0-527$ & 0 & 0.6 & - & $0-615$ \\
\hline $\begin{array}{l}\text { Leukaemia and aleukaemia } \\
(204-207)\end{array}$ & 4 & & & $52-488$ & 3 & 1.9 & 162 & $33-461$ \\
\hline Other lymphatic tissue (208) & $\begin{array}{l}4 \\
1\end{array}$ & $\begin{array}{l}2.1 \\
1 \cdot 3\end{array}$ & $\begin{array}{r}194 \\
76\end{array}$ & $\begin{array}{r}r-488 \\
2-429\end{array}$ & $\begin{array}{l}3 \\
1\end{array}$ & $\begin{array}{l}1.9 \\
1.2\end{array}$ & $\begin{array}{r}102 \\
84\end{array}$ & $\begin{array}{r}35-401 \\
2-464\end{array}$ \\
\hline Other and unspecified sites (199) & 7 & 3.8 & 184 & $74-380$ & 7 & 3.4 & 205 & $83-424$ \\
\hline Diabetes $(250)$ & 4 & 3.8 & 105 & $29-270$ & 4 & $3 \cdot 4$ & 118 & $32-301$ \\
\hline Diseases blood and blood-forming & & & & & & & & \\
\hline $\begin{array}{l}\text { organs }(280-289) \\
\text { Diseases of circulatory system }\end{array}$ & 3 & 0.7 & 444 & $88-1252$ & 1 & 0.6 & 164 & 4-929 \\
\hline $\begin{array}{l}\text { Diseases of circulatory system } \\
(390-458)\end{array}$ & 115 & $134 \cdot 7$ & 85 & $70-102$ & 106 & $121 \cdot 4$ & 87 & $71-106$ \\
\hline $\begin{array}{l}\text { Non-malignant respiratory diseases } \\
(460-519)\end{array}$ & 13 & & & $45-143$ & 11 & & 79 & $40-142$ \\
\hline Gastric and duodenal ulcer (531-534) & $\begin{array}{r}13 \\
2\end{array}$ & $\begin{array}{r}13.3 \\
1.9\end{array}$ & $\begin{array}{r}84 \\
106\end{array}$ & $\begin{array}{l}40-143 \\
13-380\end{array}$ & 11 & $\begin{array}{l}13.9 \\
1.7\end{array}$ & 118 & $\begin{array}{l}40-142 \\
14-435\end{array}$ \\
\hline Cirrhosis of the liver (571) & 5 & $7 \cdot 3$ & 69 & $22-160$ & 5 & $6 \cdot 6$ & 76 & $25-177$ \\
\hline All accidents (E800-E949) & 4 & 18.8 & 21 & $6-54$ & 4 & $17 \cdot 2$ & 23 & $6-60$ \\
\hline Suicide (E950-E959) & 8 & 6.4 & 125 & $54-246$ & 7 & 5.8 & 120 & $49-249$ \\
\hline
\end{tabular}

*Fisher's exact limits when observed less than 20, approximate limits otherwise.

†Less those exposed to arsenic, asbestos, or high levels of vinyl chloride (see text).

Table 5 Summary of myelogenous leukaemias

\begin{tabular}{|c|c|c|c|c|c|c|}
\hline Case No & Year of death & Age at death & $\begin{array}{l}\text { Year of } \\
\text { first exposure }\end{array}$ & $\begin{array}{l}\text { Year of } \\
\text { last exposure }\end{array}$ & $\begin{array}{l}\text { Years of } \\
\text { exposure }\end{array}$ & $\begin{array}{l}\text { Cumulative exposure estimate } \\
\text { (ppm months) }\end{array}$ \\
\hline $\begin{array}{l}1 \\
2 \\
3+ \\
4 \\
5\end{array}$ & $\begin{array}{l}1971 \\
1965 \\
1970 \\
1980 \\
1967\end{array}$ & $\begin{array}{l}45 \\
51 \\
72 \\
80 \\
80\end{array}$ & $\begin{array}{l}1960 \\
1950 \\
1955 \\
1943 \\
1928\end{array}$ & $\begin{array}{l}1971 \\
1952 \\
1962 \\
1962 \\
1952\end{array}$ & $\begin{array}{r}10.7 \\
1.5 \\
5.0 \\
19.5 \\
23.3\end{array}$ & $\begin{array}{r}648 \ddagger \\
18 \\
305 \\
4211 \\
343\end{array}$ \\
\hline
\end{tabular}

*Peak exposures for employees were measured in excess of $250 \mathrm{ppm}$ in some samples.

tUnderlying cause of death was classified as pneumonia, acute myelogenous leukaemia listed under other significant conditions.

†Five hundred and forty five ppm months accumulated before 1970 as reported by Ott et al,$^{6}$ and 103 ppm months accumulated during 1970 and 1971 .

In addition to the deaths from aplastic and pernicious anaemia (one each) observed by Ott et al, ${ }^{6}$ one other death occurred from diseases of blood or blood forming tissue. This man was hired in 1923 and worked in many different jobs including an area of potential arsenic exposure until 1939 when he settled into various operator jobs in alkyl benzene. He was classified as potentially exposed to several different levels of benzene including high until his retirement in 1965. His estimated career dose was $6027 \mathrm{ppm}$ months. He died in 1980 aged 80 of myelofibrosis.

The analysis of mortality in the total cohort was carried out allowing for a 15 year minimum latency period. This effectively removed those first exposed 
after 1967 from the analysis. The mortality patterns described above were not altered, although some of the cause specific SMRs were increased slightly.

Mortality results by work area are presented in table 6. Some employees worked in multiple areas, therefore they may be counted more than once in the table. Total mortality was consistently below expected levels across the three work areas. Although there were no statistically significant rises by work area, marginal excesses of skin cancer were observed only for the alkyl benzene and ethyl cellulose areas, and excess lymphopoietic cancer was observed only for the alkyl benzene area. A slight excess of lung cancer was observed among those who worked in ethyl cellulose, whereas deficits of this cancer were observed for the other two production areas. Small excesses in the category of cancer of other and unspecified sites were observed for the chlorobenzol and ethyl cellulose areas.

Table 7 presents observed and expected deaths from all cancers combined by years of exposure and interval since first exposure. Mortality was somewhat raised in the period from 15 to 19 years after first exposure; however, it was largely independent of duration of exposure.

Table 8 shows the results of dose response analyses for selected cause of death categories. Analyses were made by first lagging exposure zero years, and then 15 years to allow for the possible expression of a latency period. Duration of exposure is an important component of any cumulative dose index and is strongly correlated with survival. Failure to lag exposures to compensate for this may result in missing a dose response relation. There were no significant mortality

Table 6 Mortality summary by production area, excluding employees with competing exposures $\dagger$

\begin{tabular}{|c|c|c|c|c|c|c|}
\hline \multirow[b]{3}{*}{ Cause of death } & \multicolumn{6}{|c|}{ Production area } \\
\hline & \multicolumn{2}{|c|}{ Chlorobenzol } & \multicolumn{2}{|c|}{ Alkyl benzene } & \multicolumn{2}{|c|}{ Ethyl cellulose } \\
\hline & Obs & $\operatorname{Exp}$ & Obs & $\operatorname{Exp}$ & Obs & $\operatorname{Exp}$ \\
\hline $\begin{array}{l}\text { All causes } \\
\text { All cancer } \\
\text { Digestive system } \\
\text { Lung } \\
\text { Skin } \\
\text { Leukaemia } \\
\text { All lymphopoietic } \\
\text { Other and unspecified sites }\end{array}$ & $\begin{array}{r}44 \\
9 \\
3 \\
1 \\
0 \\
1 \\
1 \\
3\end{array}$ & $\begin{array}{r}54 \cdot 6 \\
11 \cdot 3 \\
3 \cdot 1 \\
3.9 \\
0 \cdot 2 \\
0 \cdot 4 \\
1 \cdot 1 \\
0.8\end{array}$ & $\begin{array}{r}66 \\
12 \\
4 \\
2 \\
2 \\
2 \\
4 \\
0\end{array}$ & $\begin{array}{r}74 \cdot 9 \\
15 \cdot 1 \\
4 \cdot 3 \\
4 \cdot 9 \\
0 \cdot 3 \\
0 \cdot 6 \\
1 \cdot 4 \\
1 \cdot 0\end{array}$ & $\begin{array}{r}93^{*} \\
29 \\
5 \\
11 \\
2 \\
0 \\
1 \\
4\end{array}$ & $\begin{array}{r}121 \cdot 2 \\
24 \cdot 7 \\
6 \cdot 6 \\
8 \cdot 3 \\
0 \cdot 5 \\
0.9 \\
2 \cdot 5 \\
1 \cdot 7\end{array}$ \\
\hline $\begin{array}{l}\text { Diseases blood and blood forming } \\
\text { organs } \\
\text { Diseases of circulatory system } \\
\text { Respiratory system diseases } \\
\text { Gastric and duodenal ulcer } \\
\text { Cirrhosis of liver } \\
\text { Accidents } \\
\text { Suicide }\end{array}$ & $\begin{array}{r}0 \\
25 \\
2 \\
1 \\
3 \\
1 \\
2\end{array}$ & $\begin{array}{r}0 \cdot 1 \\
27 \cdot 0 \\
3 \cdot 1 \\
0 \cdot 4 \\
1 \cdot 5 \\
3.9 \\
1 \cdot 3\end{array}$ & $\begin{array}{r}0 \\
38 \\
4 \\
1 \\
0 \\
2 \\
2\end{array}$ & $\begin{array}{r}0.2 \\
39.0 \\
4.4 \\
0.5 \\
1.9 \\
4.6 \\
1.6\end{array}$ & $\begin{array}{r}1 \\
45 \\
5 \\
0 \\
2 \\
1 \ddagger \\
3\end{array}$ & $\begin{array}{r}0.3 \\
59.5 \\
6.9 \\
0.8 \\
3.5 \\
9 \cdot 3 \\
3 \cdot 1\end{array}$ \\
\hline
\end{tabular}

* Significant at $\alpha=0.05$, two-tailed.

tLess those exposed to arsenic, asbestos, or high levels of vinyl chloride (see text).

tSignificant at $\alpha=0.01$, two-tailed.

Table 7 Observed and expected mortality from all malignant neoplasms (140-209) by years of exposure and interval since entry. Benzene cohort less exclusions*

\begin{tabular}{|c|c|c|c|c|c|c|c|c|c|c|}
\hline \multirow{2}{*}{$\begin{array}{l}\text { Years of } \\
\text { exposure }\end{array}$} & \multicolumn{10}{|c|}{ Interval since entry } \\
\hline & $\overline{0-}$ & $5-$ & $10-$ & $15-$ & $20-$ & $25-$ & $30-$ & $35-$ & $40-$ & Total \\
\hline \multirow[t]{2}{*}{$\begin{array}{c}0- \\
5- \\
10- \\
15- \\
20- \\
25- \\
30- \\
35- \\
40-\end{array}$} & $0 / 2 \cdot 3$ & $\begin{array}{l}2 / 1.9 \\
1 / 1.6\end{array}$ & $\begin{array}{l}0 / 2.4 \\
1 / 0.7 \\
2 / 1.5\end{array}$ & $\begin{array}{l}7 / 2.9 \\
2 / 0.7 \\
0 / 0.9 \\
2 / 1.2\end{array}$ & $\begin{array}{l}4 / 3 \cdot 4 \\
0 / 0 \cdot 7 \\
0 / 0.9 \\
0 / 0.6 \\
1 / 1 \cdot 1\end{array}$ & $\begin{array}{l}4 / 4.0 \\
0 / 0.6 \\
1 / 0.9 \\
1 / 0.7 \\
3 / 0.5 \\
0 / 1.0\end{array}$ & $\begin{array}{l}5 / 4 \cdot 4 \\
0 / 0 \cdot 8 \\
3 / 0 \cdot 8 \\
0 / 0 \cdot 8 \\
1 / 0 \cdot 2 \\
0 / 0 \cdot 5 \\
0 / 0 \cdot 9\end{array}$ & $\begin{array}{l}4 / 3.5 \\
0 / 1.0 \\
1 / 0.7 \\
1 / 0.6 \\
0 / 0.1 \\
0 / 0.5 \\
0 / 0.7 \\
0 / 0.4\end{array}$ & $\begin{array}{l}1 / 0.8 \\
1 / 0.5 \\
0 / 0.3 \\
0 / 0.3 \\
0 / 0.0 \\
0 / 0.2 \\
0 / 0.4 \\
0 / 0.2 \\
1 / 0.1\end{array}$ & $\begin{array}{rr}27 / 25.7 \\
5 / & 6.6 \\
7 / & 6.0 \\
4 / & 4.2 \\
5 / & 2.0 \\
0 / & 2.2 \\
0 / & 2.0 \\
0 / & 0.6 \\
1 / & 0.1\end{array}$ \\
\hline & $0 / 2 \cdot 3$ & $3 / 3 \cdot 5$ & $3 / 4 \cdot 7$ & $11 / 5.7$ & $5 / 6 \cdot 7$ & $9 / 7 \cdot 7$ & $9 / 8 \cdot 5$ & $6 / 7 \cdot 5$ & $3 / 2.7$ & $49 / 49 \cdot 2$ \\
\hline
\end{tabular}

*Less those exposed to arsenic, asbestos, or high levels of vinyl chloride (see text). 
Table 8 Mortality summary by estimated cumulative dose of benzene exposure, excluding employees with competing exposures $\dagger$

\begin{tabular}{|c|c|c|c|c|c|c|}
\hline \multirow{3}{*}{$\begin{array}{l}\text { Cause of death and } \\
\text { cumulative dose level }\end{array}$} & \multicolumn{6}{|c|}{ Exposure lagged by } \\
\hline & \multicolumn{3}{|c|}{0 years } & \multicolumn{3}{|c|}{15 years } \\
\hline & Obs & Exp & $S M R$ & Obs & Exp & $S M R$ \\
\hline \multicolumn{7}{|l|}{ All causes (001-999) } \\
\hline $\begin{array}{l}\text { 0-499 ppm months } \\
500-999 \mathrm{ppm} \text { months } \\
\geqslant \\
1000 \mathrm{ppm} \text { months } \\
\text { Total }\end{array}$ & $\begin{array}{r}115 \\
43 \\
42 \\
200\end{array}$ & $\begin{array}{r}151 \cdot 4 \\
35 \cdot 2 \\
55 \cdot 6 \\
242 \cdot 3\end{array}$ & $\begin{array}{c}76 \\
122 \\
76 \\
83^{*}\end{array}$ & $\begin{array}{r}105 \\
33 \\
27 \\
165\end{array}$ & $\begin{array}{r}117 \cdot 8 \\
25 \cdot 7 \\
37 \cdot 4 \\
180 \cdot 5\end{array}$ & $\begin{array}{r}89 \\
129 \\
72 \\
91\end{array}$ \\
\hline \multicolumn{7}{|l|}{ All cancer $(140-209)$} \\
\hline $\begin{aligned} & 0-499 \\
& 500-999 \\
& 1000 \\
& \text { Total }\end{aligned}$ & $\begin{array}{l}27 \\
11 \\
11 \\
49\end{array}$ & $\begin{array}{r}30 \cdot 0 \\
7 \cdot 3 \\
11.9 \\
49 \cdot 2\end{array}$ & $\begin{array}{r}90 \\
150 \\
92 \\
100\end{array}$ & $\begin{array}{r}27 \\
9 \\
7 \\
43\end{array}$ & $\begin{array}{r}25 \cdot 0 \\
5 \cdot 7 \\
8 \cdot 4 \\
39 \cdot 1\end{array}$ & $\begin{array}{r}108 \\
159 \\
84 \\
110\end{array}$ \\
\hline $\begin{array}{l}\text { Lung cancer }(162-163) \\
0-499\end{array}$ & 6 & $9 \cdot 7$ & 62 & 8 & $8 \cdot 6$ & 94 \\
\hline $500-999$ & 5 & $2 \cdot 5$ & 204 & 4 & $2 \cdot 0$ & 203 \\
\hline$\geqslant 1000$ & 2 & $4 \cdot 1$ & 49 & 1 & $3 \cdot 0$ & 34 \\
\hline Total & 13 & $16 \cdot 3$ & 80 & 13 & $13 \cdot 5$ & 96 \\
\hline \multicolumn{7}{|l|}{ Skin cancer (172-173) } \\
\hline $0-499$ & 4 & 0.6 & $676 \ddagger$ & 4 & 0.4 & $934 \ddagger$ \\
\hline $500-999$ & 0 & $0 \cdot 1$ & - & 0 & $0 \cdot 1$ & \\
\hline$\geqslant 1000$ & 0 & 0.2 & - & 0 & $0 \cdot 1$ & - \\
\hline \multirow{2}{*}{\multicolumn{7}{|c|}{ Lymphopoietic cancers $(200-209){ }^{4}$}} \\
\hline & & & & & & \\
\hline $0-499$ & 4 & $3 \cdot 0$ & 133 & 3 & $2 \cdot 3$ & 132 \\
\hline $500-999$ & 0 & 0.7 & - & 0 & 0.5 & - \\
\hline$\geqslant 1000$ & 2 & $1 \cdot 1$ & 183 & 2 & 0.7 & 274 \\
\hline Total & 6 & 4.8 & 125 & 5 & $3 \cdot 5$ & 142 \\
\hline \multicolumn{7}{|l|}{ Leukaemia (204-207) } \\
\hline $0-499$ & 2 & 1.2 & 167 & 2 & 0.9 & 222 \\
\hline $500-999$ & 0 & $0 \cdot 3$ & - & 0 & $0 \cdot 2$ & - \\
\hline$\geqslant 1000$ & 1 & 0.4 & 250 & 1 & $0 \cdot 3$ & 333 \\
\hline \multirow{2}{*}{\multicolumn{7}{|c|}{ Diseases of circulatory system $(390-458)$}} \\
\hline & & & & & & \\
\hline $\begin{array}{l}0-499 \\
500-999\end{array}$ & $\begin{array}{l}60 \\
22\end{array}$ & $\begin{array}{l}74 \cdot 6 \\
17 \cdot 9\end{array}$ & $\begin{array}{r}80 \\
123\end{array}$ & $\begin{array}{l}53 \\
21\end{array}$ & $\begin{array}{l}62 \cdot 6 \\
13 \cdot 7\end{array}$ & 154응 \\
\hline$\geqslant 1000$ & 24 & 28.9 & 83 & 15 & $20 \cdot 1$ & 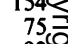 \\
\hline Total & 106 & $121 \cdot 4$ & 87 & 89 & $96 \cdot 3$ & $92 \frac{0}{\Xi}$ \\
\hline
\end{tabular}

*Significant at $\alpha=0.05$.

tLess those exposed to arsenic, asbestos, or high levels of vinyl chloride (see text).

$\ddagger$ Significant at $\alpha=0.01$.

trends for any cause of death evaluated. Oddly enough, mortality from several causes was somewhat raised among those exposed from 500 to $999 \mathrm{ppm}$ months. All four skin cancers were observed to have been in the lowest dose cateory.

\section{Discussion}

Mortality was updated to the end of 1982 for 594 benzene exposed employees studied originally by Ott et $a l,{ }^{6}$ and for an additional 362 exposed workers not studied previously. Total mortality was observed to have been significantly below the comparable United States population but was consistent with the general pattern for other employees at this company location. ${ }^{12}$ Presumably this is due to initial selection for work based on good health, the benefits which accrue from continued employment, and the absence of a major occupational hazard. Two additional deaths from leukaemia were observed in the cohort, whereas one additional death from this cause was expected. Taken together with the two deaths from leukaemia previously reported for this cohort, the total still does not represent a statistically significant excess of total leukaemia. It does represent, however, a significant excess of mortality from myelogenous leukaemia (4 observed versus 0.9 expected; $p=0.011$ ) when mortality data compiled from the Surveillance, Epidemiology and End Results programme are used as a comparison. ${ }^{14}$

The death from myelofibrosis is interesting since this condition is believed to represent a primary overgrowth of a native fibroblastic cell line, analogous to myelogenous leukaemia. ${ }^{15}$ There have been several reports of myelofibrosis among employees with allegedly heavy benzene exposure (B D Goldstein, unpublished data). Among the deaths from other lymphoreticular cancer was one multiple myeloma. This did not represent an excess over expectation and is notable only because Decoufle et al suggested a link between this condition and benzene. ${ }^{3}$

An unexpected finding was a significant excess of 
mortality from skin cancer, based on four observed cases. A review of the death certificates showed that three of the four were malignant melanomas, the fourth was described as a squamous cell carcinoma. There were no unusual or common characteristics among the affected individual's work histories that would suggest a link with benzene or any other workplace exposures. All four were classified into the lowest cumulative dose category. Previous reports of mortality among benzene exposed workers have not noted excess skin cancer, or more specifically melanoma, and thus its finding here may represent an isolated or chance occurrence ${ }^{1-4}$ (B D Goldstein and O Wong, unpublished data).

Mortality from accidental causes of death was observed to have been substantially below expectation, even by indirect comparison with other employees from this location. ${ }^{12}$ There is no available explanation for this deficit but again it may represent a chance occurrence.

Analysis by work area, duration of exposure, and cumulative dose index did not show patterns suggestive of a causal association between exposure to benzene and any particular category of death. Three of the four patients with leukaemia, one with multiple myeloma, and one with myelofibrosis worked in alkyl benzene. This may indicate that some additional cofactor may be important in the aetiology of these deaths. Other materials present in this work area included ethylbenzene, divinylbenzene, toluene, xylene, isopropyl benzene, styrene, diisopropyl benzene, and alpha-methyl styrene.

Askoy recently suggested a link between exposure to benzene and lung cancer. ${ }^{2}$ We found no evidence to support such an association in our study. Lung cancer was not found to be excessive in the total cohort or in the cohort less exclusions (13 observed against 16.3 expected), nor was there a trend of increasing lung cancer risk with increasing exposure. The study had $95 \%$ power to detect a twofold risk of lung cancer with a one tailed test and alpha $=0.05$, and $57 \%$ power to detect a one and a half-fold risk.

Although this study provides support for the association between exposure to benzene and acute myelogenous leukaemia reported by others ${ }^{1}$ (B D Goldstein), several factors complicate use of these data for risk assessment. These include the problem of a small number of events, competing exposures to other potentially hazardous materials, and the uncertain contribution of unquantified brief exposures.

We gratefully acknowledge the technical support received from Dr M Gerald Ott, Karen Wymer, Janice Cartmill, and Bernard Lasich. Angie Grzegorczyk typed the manuscript.

\section{References}

1 International Agency for Research on Cancer. Monographs on the evaluation of carcinogenic risks of chemicals to humans. Vol 29. Some industrial chemicals and dyestuffs. Geneva: IARC, 1982:93-148.

2 Askoy M. Malignancies due to occupational exposures to benzene. Am J Ind Med 1985;7:395-402.

3 Decoufle P, Blattner WA, Blair A. Mortality among chemical workers exposed to benzene and other agents. Environ Res 1983;30:16-25.

4 Infante PF, White MC. Projections of leukemia risk associated with occupational exposure to benzene. Am J Ind Med 1985;7:403-14.

5 United States Environmental Protection Agency. National emission standards for hazardous air pollutants. Regulation of benzene. Fed Reg 1984;49:23488.

6 Ott MG, Townsend JC, Fishbeck WA, Langner RR. Mortality among individuals occupationally exposed to benzene. Arch Environ Health 1978;33:3-10.

7 Monson RR. Analysis of relative survival and proportional mortality. Computers and Biomedical Research 1974;7:325-32.

8 Fisher RA. Statistical methods and scientific inference. London: Oliver and Boyd, 1956.

9 Rothman KJ, Boice JD Jr. Epidemiologic analysis with a programmable calculator. Boston: Epidemiology Resources, 1982.

10 Breslow NE, Lubin JH, Marek P, Langholtz B. Multiplicative models and the analysis of cohort data. Journal of the American Statistical Association 1983;78:1-12.

11 Ott MG, Holder BB, Gordon H. Respiratory cancer and occupational exposure to arsenicals. Arch Environ Health 1974;29:250-5.

12 Ott MG. Effects of selection and confounding on mortality in an occupational cohort. Ann Arbor: University Microfilms International, 1983. (PhD dissertation.)

13 Ott MG, Langner RR, Holder BB. Vinyl chloride exposure in a controlled industrial environment. Arch Environ Health 1975;30:333-9.

14 Surveillance, Epidemiology and End Results (SEER). Incidence and mortality, 1973-1977. Washington: US Government Printing Office, 1981. National Cancer Institute monograph 57.

15 Robbins SL, Angell M, Kumar V. Basic pathology. 3rd ed. Philadelphia: WB Saunders, 1981. 